\title{
Cervical Adenoid Basal Carcinoma: A Case Report
}

\author{
Asuman Argon ${ }^{1}$, Alper Şener ${ }^{2}$, Osman Zekioğlu², Coşan Terek ${ }^{3}$ Necmettin Özdemir² \\ ${ }^{1}$ Clinic of Medical Pathology, Ministry of Health Izmir Bozyaka Training and Research Hospital, Izmir, Turkey \\ ${ }^{2}$ Department of Pathology, Faculty of Medicine, Ege University, Izmir, Turkey \\ ${ }^{3}$ Department of Gynecology and Obstetrics, Faculty of Medicine, Ege University, Izmir, Turkey
}

\begin{abstract}
Adenoid basal carcinoma $(A B C)$ is a rare epithelial tumor of the cervix. It makes up approximately $1 \%$ of all cervical adenocarcinomas. Rare cases have been associated with common cervical epithelial tumors. We present a case of ABC associated with typical squamous cell carcinoma. A 54-year-old postmenopausal woman underwent D\&C for vaginal bleeding. Histologically, the tumor was characterized by small cells with a narrow cytoplasm, making up islands and cords. Peripheral palissading in the cells surrounding the cystic areas that contained central cellular debris and keratin was noted. The patient underwent total hysterectomy, bilateral salpingo-oophorectomy, pelvic lymphadenectomy and omentectomy. Large cell keratinized type squamous cell carcinoma areas in the cervix were noted besides the limited ABC areas. After surgery, the patient was treated with radiation therapy. A retroperitoneal metastasis was found on the first year and chemotherapy was administered. The patient has no evidence of disease 27 months after the first diagnosis. $\mathrm{ABC}$ makes up quite a rare group of cervical cancers and should be kept in mind during the evaluation so that a differentiation can be made with tumors with similar morphology as it can show various histological patterns, and can be seen together with more aggressive cancers.
\end{abstract}

Key Word: Adenoid basal carcinoma, HPV, cervix, squamous cell carcinoma

\section{Introduction}

Adenoid basal carcinoma was first defined by Baggish and Woodruff in 1966 (1).It makes up less than $1 \%$ of cervical adenocarcinomas and fewer than 100 cases have been reported $(2,3)$. It has usually been defined in postmenopausal women as one of the asymptomatic tumors that do not cause an abnormal appearance in the cervix (4). Cviko et al. (5) reported four different components of the tumor in their article. These were high grade squamous intraepithelial neoplasic areas; an invasive squamous component surrounded by basal cells and with marked maturation in the center; small basaloid islands that spread to the periphery; and small acini observed without columnar cell differentiation in these islands. It has been reported that $A B C$ can rarely be seen concurrently with typical squamous cell carcinoma or even mixed epithelial/ mesenchymal tumors (6-8). The prognosis is quite good in pure cases, while it is defined by the accompanying tumor in mixed cases (9).This carcinoma must be distinguished from the more aggressive adenoid cystic carcinoma (ACC) of the uterine cervix and they are sometimes very similar histologically. $A B C$ can usually be distinguished from $A C C$ by the lack of the characteristic intraluminal hyaline material frequently present in ACC; the presence of smaller, less pleomorphic nuclei; and less mitotic activity than is characteristic of ACC (10). We herein describe a case of adenoid basal carcinoma in the cervix which is a very rare type of cervical cancer and its differentiation is important from the more aggressive cancers that it is morphologically similar to.

\section{Case Report}

A 54-year-old female who had been postmenopausal for 3 years presented at the obstetrics and gynecology department because of vaginal bleeding. She was gravida 3, parity 2 and there was nothing of significance in the personal or family history. The laboratory evaluation results were CEA $7.2 \mathrm{ng} /$ $\mathrm{mL}, \mathrm{CA} 19-936 \mathrm{IU} / \mathrm{mL}$ and no other abnormal findings were detected. Vaginal palpation revealed a $4 \mathrm{~cm}$ tumoral mass at the isthmic level. The speculum examination showed a dilated cervix and the necrotic tumor mass was protruding out of the cervical canal. Computerized tomography showed a normal uterus and ovaries, irregularity at the periuterine fatty tissue layers and occasional millimetric lymph nodes. The case underwent diagnostic curettage instead of cervical biopsy or conization because the tumor was necrotic and protruding out of the cervical canal.

The curettage material, about $2 \mathrm{cc}$ in volume, was fixed in formalin and, after routine processing, embedded in paraffin and was evaluated with the light microscope after 3-micronthick sections were made. We used Vimentin, monoclonal CEA and Cytokeratin 5/6 for the immunohistochemical examinations and HPV6 and HPV16 for the chromogenic in situ hybridization (CISH) method.

Histological examination revealed that the tumor was characterized by islands and cords that had developed through the proliferation of small basaloid cells. The tumor cells were small and uniform, with dark oval nuclei without conspicuous nucleoli and scanty cytoplasm. Mitotic activity was low and no 
atypical mitosis was seen. The central portions of the tumor cell nests formed cystic spaces filled with necrotic debris or eosinophilic material or occasionally showed squamous differentiation. The tumor showed an infiltrative growth pattern but there was no desmoplasia or lymphocytic response. The case received a diagnosis of "Adenoid Basal Carcinoma" and the patient underwent total type 1 hysterectomy, bilateral salpingo-oophorectomy, pelvic lymphadenectomy and omentectomy. Although omentectomy is not a routine procedure for cervical cancer, it led us to make complete staging procedure in this rare tumor. Macroscopic evaluation of the histological material revealed a necrotic tumor that showed occasional bleeding with a dirty yellow sectional surface $2.2 \times 1.9 \times 1 \mathrm{~cm}$ in size that had dilated the cervix to a barrel-shape. There was $1 / 2$ external $(19 / 20 \mathrm{~mm})$ stromal invasion in the tumor. Approximately $40 \%$ large cell keratinized type squamous cell carcinoma together with approximately $60 \% A B C$ were observed in the histological examination (Figure 1). Various components were noted inside the basaloid tumor. The first of these components was the invasive squamous component with basal cells at the periphery and maturation at the center (Figure 2), while the second was small acini seen without columnar cell differentiation in the tumor islands (Figure 3) and the last one was basaloid islands spreading to the periphery. We encountered necrobiotic debris in the lumen-type cystic spaces but there was no hyaline material. We also observed moderate pleomorphism and rare mitosis. Neither lymphatic nor vascular invasion was identified. There was no tumor in the lymph nodes, omentum or preoperative abdominal lavage fluid.

In order to further elucidate the nature of the tumor, immunohistochemical stains were performed. The basaloid cells were positive for m-CEA and Vimentin while the cells with squamous differentiation and those around the acini were negative. Cytokeratin 5/6 showed strong positive staining in the basaloid cells but weak and focal staining in the other cells. The chromogenic in situ hybridization (CISH) method showed that the tumor cells were positive for HPV-6 and HPV 16.

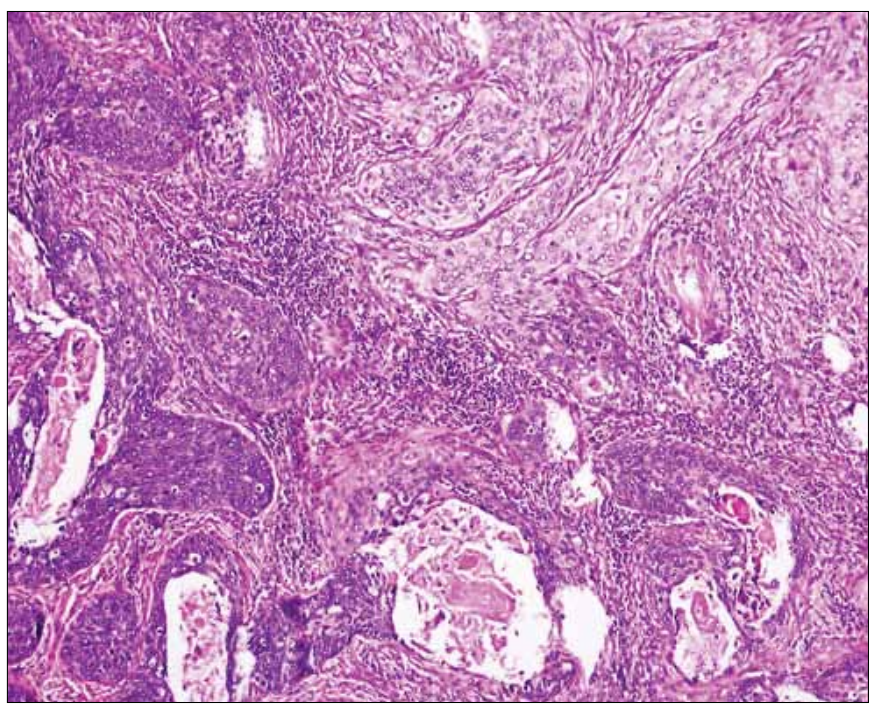

Figure 1. Large cell keratinized type squamous cell carcinoma together with ABC. Hematoxylin- Eosin, X10
After surgery, the patient was treated with radiation therapy. The patient, who received the radiotherapy treatment in a different center, presented with a complaint of right upper quadrant pain during the first postoperative year and radiological evaluations revealed a $6 \times 5 \mathrm{~cm}$ hypervascular solid mass that infiltrated the fatty tissue at the Morrison level, localized between the middle pole of the right kidney and the $6^{\text {th }}$ segment of the liver. The retroperitoneal mass was excised together with the right adrenal gland on laparotomy. Histopathologically, we found squamous cell carcinoma and adenoid basal carcinoma metastasis. The patient received chemotherapy and the clinical and radiological follow-up is continuing. She is currently alive at the postoperative $27^{\text {th }}$ month and no recurrence and/or other metastasis have been detected.

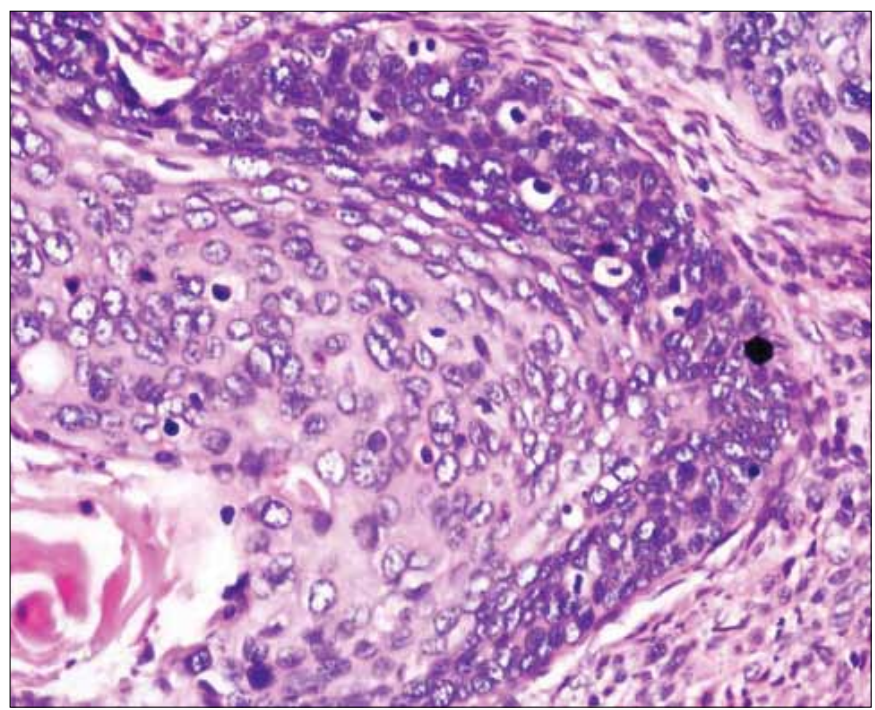

Figure 2. The invasive squamous component with basal cells at the periphery and maturation at the center. Hematoxylin- Eosin, X40

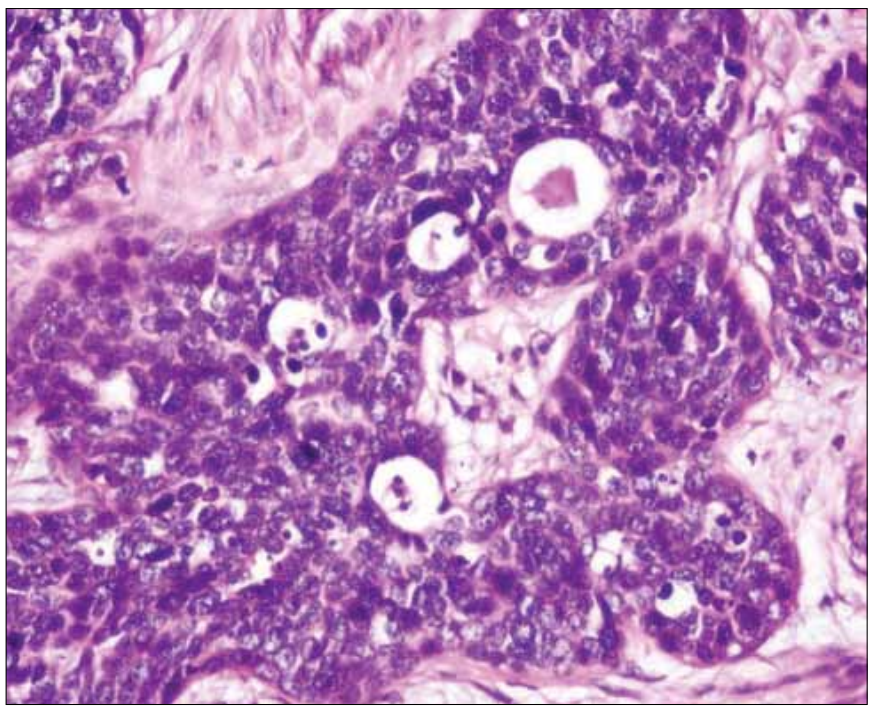

Figure 3. Small acini without columnar cell differentiation in the tumor islands. Hematoxylin- Eosin, X40 


\section{Discussion}

Adenoid basal carcinoma of the uterine cervix, first distinguished from adenoid cystic carcinoma by Baggish and Woodruff in 1966, accounts for less than $1 \%$ of all cervical adenocarcinomas, and fewer than 100 cases have been reported (1-3). It is frequently seen in postmenopausal women, as in this article, but cases under 40 years old have also been reported in recent years $(3,4,11)$.

The patients are usually asymptomatic and have come to medical attention after an abnormal cervical smear result. Symptoms, if any, are usually in the form of vaginal bleeding, as in this article, while cervical prolapse and abdominal mass can be seen less commonly (12).

The cellular origin is not known clearly but the authors who were the first to define the condition put forward two hypotheses. The first one is that the tumor develops from the multipotent reserve cell layer of the cervical epithelium that can show squamous or glandular differentiation. The second hypothesis, which is quite suspect and speculative, is that it develops from heterotopic ectodermal components in the cervix. The main basis of these hypotheses is the morphological similarity of the cases with adenosquamous (especially for the first hypothesis) and dermal basal cell carcinoma (especially for the second hypothesis) (12). However, the second hypothesis has not been accepted in the following years and most evidence has pointed to $A B C$ developing from cells that can show pluripotent differentiation. This evidence is that these tumors can be seen in association with various tumors such as squamous, glandular, biphasic epithelial (adenosquamous), biphasic epithelial/mesenchymal (carcinosarcomas) differentiation and they can contain squamous differentiation and pseudoglandular structures, with both features seen in our case. Finally, ABC cells have been shown to contain a notched oval-round nucleus, widespread organelles and intracytoplasmic filaments, similar to cervical reserve cells (13).

The tumor has been reported to contain up to four different histological appearances: areas of high grade squamous intraepithelial neoplasia; invasive squamous areas containing marked maturation in the center and basal cells in the periphery; basaloid islands that spread to the periphery and small acini without columnar cell differentiation in these islands (5). The first component was not seen in our case but the other three histological appearances were present in various ratios.

Senzaki et al. (12) report that immunohistochemical stains show the palissading basaloid cells to be positive for cytokeratin $7,8,14,17,18,19$, the foci of squamous differentiation to be positive for cytokeratin 10 and 13 , and the cells surrounding the lumina to be positive for cytokeratin 7, 8, 18, 19 and CEA. The same article reports that positive results for $p 53$ are rare, while there is no staining with estrogen receptor, progesterone receptor, S-100, Actin and CA125. The tumor has also been reported to be EMA positive but Type IV collagen and laminin negative immunohistochemically $(7,9)$. We found m-CEA and Vimentin were positive in basaloid cells while negative in the cells seen around acini showing squamous differentiation. There was strong positive staining in basaloid cells with cytokeratin $5 / 6$ while staining in the other cells was weak and focal.
Studies investigating the molecular features of adenoid basal carcinoma have reported the lack of K-ras-2 point mutations, while p53 point mutations thought to be associated with a worse prognosis were found in some cases and HPV concurrence in all cases $(14,15)$. The role of HPV in $A B C$ pathogenesis has been shown in many studies $(5,7,8,11$, $14,15)$. Our case was also positive for HPV and HPV 16 with the $\mathrm{CISH}$ method, as in the other cases in the literature.

The differential diagnosis of adenoid basal carcinoma includes all malignancies with a basaloid morphology located in the cervix (for example: ACC, basaloid variant of squamous carcinoma, neuroendocrine carcinomas, carcinosarcomas, etc.). However, the malignancy that should primarily be considered for differentiation is ACC, which is quite similar morphologically but is expected to have a more aggressive course $(4,10)$. ACC is defined with irregular polypoid masses that frequently bleed, in contrast to $A B C$. Both tumors have a lumen structure and squamous differentiation areas but cellular pleomorphism, mitosis, necrosis, stromal hyalinization, perineural and lymphovascular invasion and metastasis are expected to be more frequent in $A C C$ than $A B C$. Immunohistochemical stains show both tumors to be positive for CEA, EMA, CK 7. However, a strong positive result for Type IV collagen and laminin is only expected in ACC. Finally, it should be noted that these tumors can also be found together $(9,12)$.

The cell nests of conventional squamous cell carcinoma with basaloid features may be of a similar morphology to ABC. However, cell nests of $A B C$ must be predominantly small and blandappearing and cytological atypia is less noticeable in comparison with conventional squamous cell carcinoma $(8,10)$.

Pure $A B C$ is known to have a quite favorable prognosis. Some authors suggest using "adenoid basal epithelioma" or "adenoid basal tumor" to define the pathology as the term "carcinoma" may cause a social, psychological and financial burden for the patient. Radical surgery is adequate for the treatment of pure cases. However, the prognosis is determined by the accompanying malignancy in mixed cases (9). Our case had an accompanying squamous cell carcinoma and a retroperitoneal metastasis was found in the first year.

\section{Conclusion}

Adenoid basal carcinoma makes up quite a rare group of cervical cancers and should be kept in mind during the evaluation so that a differentiation can be made with tumors with similar morphology as it can show various histological patterns and can be seen together with more aggressive cancers.

\section{Conflict of Interest}

No conflict of interest was declared by the authors.

\section{References}

1. Baggish MS, Woodruff JD. Adenoid-basal carcinoma of the cervix. Obstet Gynecol 1966;2:213-8.

2. Baggish MS, Woodruff JD. Adenoid basal lesions of the cervix. Obstet Gynecol 1971;6:807-19. 
3. DePond WD, Flauta VS, Lingamfelter DC, Schnee DM, Menendez KP. Adenoid basal carcinoma of the cervix in a 20 -year-old female: a case report. Diagn Pathol 2006;1:20. [CrossRef]

4. Ferry JA, Scully RE. "Adenoid cystic" carcinoma and adenoid basal carcinoma of the uterine cervix. A study of 28 cases. Am J Surg Pathol 1988;2:134-44. [CrossRef]

5. Cviko A, Briem B, Granter SR, Pinto AP, Wang TY, Yang YC, et al. Adenoid basal carcinomas of the cervix: a unique morphological evolution with cell cycle correlates. Hum Pathol 2000;6:740-4. [CrossRef]

6. Mills, Stacey E. Sternberg's Diagnostic surgical pathology. Philadelphia: Lippincott Williams \& Wilkins, 2010 (Nucci R.M, Crum C.P,ed. The Cervix)

7. Grayson W, Taylor LF, Cooper K. Carcinosarcoma of the uterine cervix: a report of eight cases with immunohistochemical analysis and evaluation of human papillomavirus status. Am J Surg Pathol 2001;3:338-47. [CrossRef]

8. Parwani AV, Smith Sehdev AE, Kurman RJ, Ronnett BM. Cervical adenoid basal tumors comprised of adenoid basal epithelioma associated with various types of invasive carcinoma: clinicopathologic features, human papillomavirus DNA detection, and P16 expression. Hum Pathol 2005;1:82-90. [CrossRef]

9. Russell MJ, Fadare O. Adenoid basal lesions of the uterine cervix: evolving terminology and clinicopathological concepts. Diagn Pathol 2006;1:18. [CrossRef]
10. Kurman RJ, Ellenson LH, Ronnett BM. Blaunstein's Pathology of the Female Genital Tract. New York: Springer 2011(Kurman RJ, ed. Adenoid basal carcinoma)

11. Zámecník M, Skrivánek A. Adenoid basal epithelioma of the uterin cervix in 21-year-old patient. Report of a case with histologic and immunohistochemical study. Cesk Patol 2005;4: 157-62.

12. Senzaki H, Osaki T, Uemura Y, Kiyozuka Y, Ogura E, Okamura $A$, et al. Adenoid basal carcinoma of the uterine cervix: Immunohistochemical study and literature review. Jpn J Clin Oncol 1997;6:437-441. [CrossRef]

13. Hiroi M, Fukunaga T, Miyazaki E, Hayashi $Y$, Kuroda $N$, Toi M, et al. Adenoid basal carcinoma of the uterine cervix: a case report with ultrastructural findings. Med Electron Microsc 2000;33: 241-5. [CrossRef]

14. Jones MW, Kounelis S, Papadaki H, Bakker A, Swalsky PA, Finkelstein SD. The origin and molecular characterization of adenoid basal carcinoma of the uterine cervix. Int J Gynecol Pathol 1997;4:301-6. [CrossRef]

15. Takeshima Y, Amatya VJ, Nakayori F, Nakano T, Iwaoki Y, Daitoku $\mathrm{K}$, et al. Co-existent carcinosarcoma and adenoid basal carcinoma of the uterine cervix and correlation with human papillomavirus infection. Int J Gynecol Pathol 2002;2: 186-90. [CrossRef] 